\title{
Downregulated Mucin 1 alleviates paclitaxel resistance in non-small cell lung cancer cells
}

\author{
HONGYU XU ${ }^{1,2}$, HUI GAO ${ }^{1}$, HUA LI $^{1}$, DONG LI ${ }^{1}$, WEIWEI YUAN ${ }^{1}$, LING ZHANG ${ }^{1}$, PENG CHENG $^{1}$, \\ XIAOMEI SU ${ }^{1}$, ZHIHUI LI ${ }^{1}$, GUANGJIE WANG ${ }^{1}$ and TAO ZHANG ${ }^{1}$ \\ ${ }^{1}$ Department of Oncology, The General Hospital of Western Theater Command, Chengdu, Sichuan 610083; \\ ${ }^{2}$ Department of Oncology, AVIC 363 Hospital, Chengdu, Sichuan 610041, P.R. China
}

Received June 10, 2019; Accepted January 14, 2020

DOI: $10.3892 / \mathrm{mmr} .2020 .11349$

\begin{abstract}
Multidrug resistance of non-small cell lung cancer (NSCLC) is a common clinical problem, which is one of the main reasons leading to the failure of chemotherapy. Therefore, how to overcome or prevent drug resistance has become a hot and difficult issue in clinical research. The present study was designed to investigate the expression patterns, functions and underlying mechanisms of MUC1 in regulating paclitaxel-resistant cell line A549/PR in NSCLC. RT-qPCR and western blot was performed to determine the mRNA and protein level, respectively. CCK-8 was conducted to determine the cell viability of A549/PR cells. Moreover, flow cytometry assay was applied to examine the apoptosis rate of A549/PR. Herein, the MUC1 was over-expressed in clinic NSCLC tissues and A549/PR cells. Silence of MUC1 could obviously suppress the proliferation and promote apoptosis of A549/PR cells in treatment of paclitaxel through up-regulating the expression of Bax and Caspase-3, and down-regulating the expression of $\mathrm{Bcl}-2$, suggesting that chemotherapy combined with the modulation of MUC1 might be characterized as a promising therapeutic approach to overcome paclitaxel-resistance in NSCLC in the future.
\end{abstract}

\section{Introduction}

Lung cancer has the highest morbidity and mortality of all malignant tumors and is a serious threat to human health. Non-small cell lung cancer (NSCLC) accounts for $80 \%$ of all lung malignancies and is the main pathological type of lung cancer (1). NSCLC has a young age of onset and is characterized by increasing morbidity and mortality rates. The current treatment strategies for patients with NSCLC include

Correspondence to: Dr Tao Zhang, Department of Oncology, The General Hospital of Western Theater Command, 270 Rongdu Road, Chengdu, Sichuan 610083, P.R. China

E-mail: zhangtao269@126.com

Key words: non-small cell lung cancer, MUC1, paclitaxel resistance, proliferation, apoptosis surgery, radiotherapy, immunotherapy and chemotherapy, as well as a combination of these treatment modalities (2). With the development of imaging techniques and molecular biology techniques, great progress has been made in the early diagnosis and treatment of patients with NSCLC. Of note, epidermal growth factor receptor (EGFR)-tyrosine kinase inhibitors may be used to treat patients with NSCLC with EGFR mutations, and have been demonstrated to improve prognosis and prolong the survival time of the aforementioned patients (3). However, the 5-year survival rate of patients with NSCLC remains low and the 5-year recurrence rate following complete surgical resection of stage I NSCLC is as high as $50 \%$. The majority of patients are diagnosed in the advanced stages of the disease, and present with even higher recurrence and metastasis rates (4).

Certain biological indicators, including histological and tumor-node-metastasis staging, number of lymph node metastases and molecular immune markers, such as EGFR mutation status, currently serve as prognostic predictors and guide treatment strategies in patients with NSCLC (5). However, chemotherapy resistance is one of the key factors leading to poor prognosis in patients with NSCLC and remains a challenging clinical problem $(6,7)$. Despite surgical intervention, radiotherapy and targeted therapies, the majority of patients require standardized treatment with paclitaxel. Paclitaxel is a type of taxane, and exerts its cytotoxic effect predominantly by disrupting microtubule assembly. Paclitaxel inhibits microtubule depolymerization and leads to cell cycle arrest in the $\mathrm{G} 2 / \mathrm{M}$ phase, resulting in apoptosis or necrosis of tumor cells $(8,9)$. However, in clinical treatment, the majority of patients develop resistance to paclitaxel chemotherapy, which eventually leads to treatment failure and affects the survival time of patients $(10,11)$. The mechanisms of paclitaxel resistance are multifaceted and complex, and include changes in cell membrane dynamics, increased drug metabolism, alterations in DNA repair mechanisms, cell cycle dysregulation and decreased apoptosis and autophagy $(12,13)$. Therefore, investigating the mechanisms of paclitaxel resistance may improve the outcome of patients with NSCLC.

Improvements in molecularbiology techniques have allowed the identification of oncogenes and tumor suppressor genes, which are an area of current interest in lung cancer research and may provide a valuable basis for individualized treat- 
ment and prognosis evaluation of patients with NSCLC (14). Mucin 1 (MUC1) is a member of the membrane-bound mucin family of glycoproteins. MUC1 is located in the apical surface of epithelial cells in the mammary glands, pancreas and gastrointestinal, respiratory and urogenital tracts, and under physiological conditions is not detected by the immune system. MUC1 is characterized by polar expression, apical distribution and rich glycosylation (15). MUC1 is ubiquitously expressed on the cell surface in several types of cancer, including esophageal and gastric cancer. Furthermore, MUC1 loses polarity and become hypoglycosylated in cancer cells and may serve as a diagnostic marker or a therapeutic target $(16,17)$. MUC1 is spontaneously hydrolyzed to produce two subunits, the $\alpha$ and $\beta$ subunits. The $\alpha$ subunit, also known as MUC1-N, is characterized by 20-200 variable number of tandem repeats (VNTRs), which are serine-, threonine- and proline-rich and may be modified by $\mathrm{O}$-glycosylation. The $\beta$ subunit, also known as MUC1-C, is composed of MUC1-extracullar domain, MUC1-transmembrane and MUC1-cytoplasmic tail (CT). MUC1-CT is highly conserved and can interact with phosphoinositide 3-kinase (PI3K), C-terminal Src kinase (CSK) and nuclear factor $\kappa \mathrm{B}(\mathrm{NF}-\kappa \mathrm{B})$ signaling pathways to regulate cell activity (18-20). Previous studies have reported that MUC1 is upregulated in several types of cancer, such as esophageal squamous-cell carcinoma $(16,21)$. The high expression level of MUC1 in lung cancer has been closely correlated with early recurrence, poor prognosis and a high metastatic potential (22). Furthermore, MUC1 knockdown suppressed lung cancer growth and invasion by inhibiting cell proliferation and inducing apoptosis (23). However, despite these findings, the association between MUC1 and paclitaxel resistance in patients with NSCLC remains unclear. Therefore, the aim of the present study was to explore the role of MUC1 in paclitaxel-resistant lung cancer cell line A549/PR, and to investigate the associated mechanism.

\section{Materials and methods}

NSCLC tissue collection. A total of 30 patients with NSCLC patients were recruited at The General Hospital of Western Theater Command from February 23, 2016 to January 1, 2018. Tumor and adjacent non-cancerous tissues were collected, snap frozen in liquid nitrogen and stored at $-80^{\circ} \mathrm{C}$. The present study was approved by the Ethics Committee of The General Hospital of Western Theater Command and all patients provided written informed consent.

Cell culture and transfection. A549 cells were purchased from the Shanghai Institute of Biochemistry and Cell Biology, Chinese Academy of Sciences, and A549/PR cells were purchased from Nanjing KeyGen Biotech, Co., Ltd. The two cell types were cultured in Dulbecco's modified Eagle medium (DMEM; Gibco; Thermo Fisher Scientific, Inc.) supplemented with $10 \%$ fetal bovine serum (FBS; Invitrogen; Thermo Fisher Scientific, Inc), $100 \mathrm{U} / \mathrm{ml}$ penicillin and $100 \mu \mathrm{g} / \mathrm{ml}$ streptomycin (Invitrogen; Thermo Fisher Scientific, Inc.). All cells were maintained in a humidified atmosphere containing $5 \% \mathrm{CO}_{2}$ at $37^{\circ} \mathrm{C}$.

Paclitaxel-resistant NSCLC cell lines A549/PR were treated with $0.5 \mu \mathrm{M}$ of paclitaxel (Glpbio) in the culture for
3 months (24). Then cells were maintained in $1 \mu \mathrm{M}$ of PTX to maintain this drug-resistant phenotype. Cellular assays were carried out when the cells were in the logarithmic growth phase.

Two small interference (si) RNAs targeting MUC1 and a scrambled siRNA were designed and synthesized by Ribobio. The $50 \mathrm{nM}$ siRNAs were transfected into A549/PR cells $\left(5 \times 10^{4}\right.$ cells $)$ using Lipofectamine ${ }^{\circledR} 2000$ reagent (Thermo Fisher Scientific, Inc.) according to the manufacturer's instructions when cells reached $50 \%$ confluence. The sequences of MUC1 siRNA and its NC were as follows: MUC1 siRNA, 5'-AAGACTGATGCCAGTAGCACT-3'; NC, 5'-AATTCTCCGAACGTGTCACGT-3'. After 72 h transfection, reverse-transcription quantitative PCR (RT-qPCR) and western blotting were used to assess the silence effect.

Cell proliferation assay. Cell proliferation was evaluated using the Cell Counting Kit-8 assay (CCK-8; Dojindo Molecular Technology). Briefly, A549/PR cells transfected with MUC1 siRNA or NC siRNA were cultured in a 96-well plate $\left(3 \times 10^{3}\right.$ cells/well). The cells were incubated for $0,12,24$, 48 or $72 \mathrm{~h}$ at $37^{\circ} \mathrm{C}$ and a total of $10 \mu \mathrm{l}$ CCK- 8 solution was added per well. The cells were subsequently incubated for $4 \mathrm{~h}$ and the optical density at a wavelength of $450 \mathrm{~nm}$ was measured using a microplate reader (Peiou).

Cell apoptosis analysis. Cell apoptosis was analyzed with an Annexin V Apoptosis Detection kit I (BD Biosciences). A total of $48 \mathrm{~h}$ following transfection, A549/PR cells were digested and washed twice in pre-chilled PBS. The cells were subsequently incubated with fluorescein isothiocyanate annexin $\mathrm{V}$ and propidium iodide for $15 \mathrm{~min}$ in the dark. Apoptosis was analyzed using a flow cytometer (FACScan; BD Biosciences) using FlowJo v7.6.1 (FlowJo LLC).

$R T$ - $q P C R$. At $48 \mathrm{~h}$ post-transfection, the total RNA was extracted from tissue specimens and transfected A549/PR cells using TRIzol reagent (Invitrogen; Thermo Fisher Scientific, Inc.) according to the manufacturer's instructions. RNA was reversed transcribed into cDNA using the TaqMan Reverse Transcription kit (Applied Biosystems; Thermo Fisher Scientific, Inc.). qPCR was subsequently performed using the Fast Start Universal SYBR Green Master mix (Roche Applied Science) and a 7500 Real-time system (Applied Biosystems; Thermo Fisher Scientific, Inc.). The following primers were used: MUC1 forward, 5'-TCCTTTCTCTGCCCAGTCTG-3' and reverse, 5'-GTGTGGTAGGTGGGGTACTC-3', GAPDH forward, 5'-GGACCTGACCTGCCGTCTAG-3' and reverse, 5'-GTAGCCCAGGATGCCCTTGA-3'. GAPDH was used as the reference gene.

Western blotting. At $48 \mathrm{~h}$ post-transfection, the total protein was extracted from tissue specimens and transfected A549/PR cells according to the manufacturer's protocol. Total protein was quantified using a bicinchoninic acid assay kit (Vazyme) and $50 \mu \mathrm{g}$ protein/lane was separated via SDS-PAGE on a $10 \%$ gel. The separated proteins were subsequently transferred onto polyvinylidene difluoride membranes and blocked in $5 \%$ skim milk in TBST for $1.5 \mathrm{~h}$ at $25^{\circ} \mathrm{C}$. The membranes were incubated with primary antibodies against MUC1 (cat. 


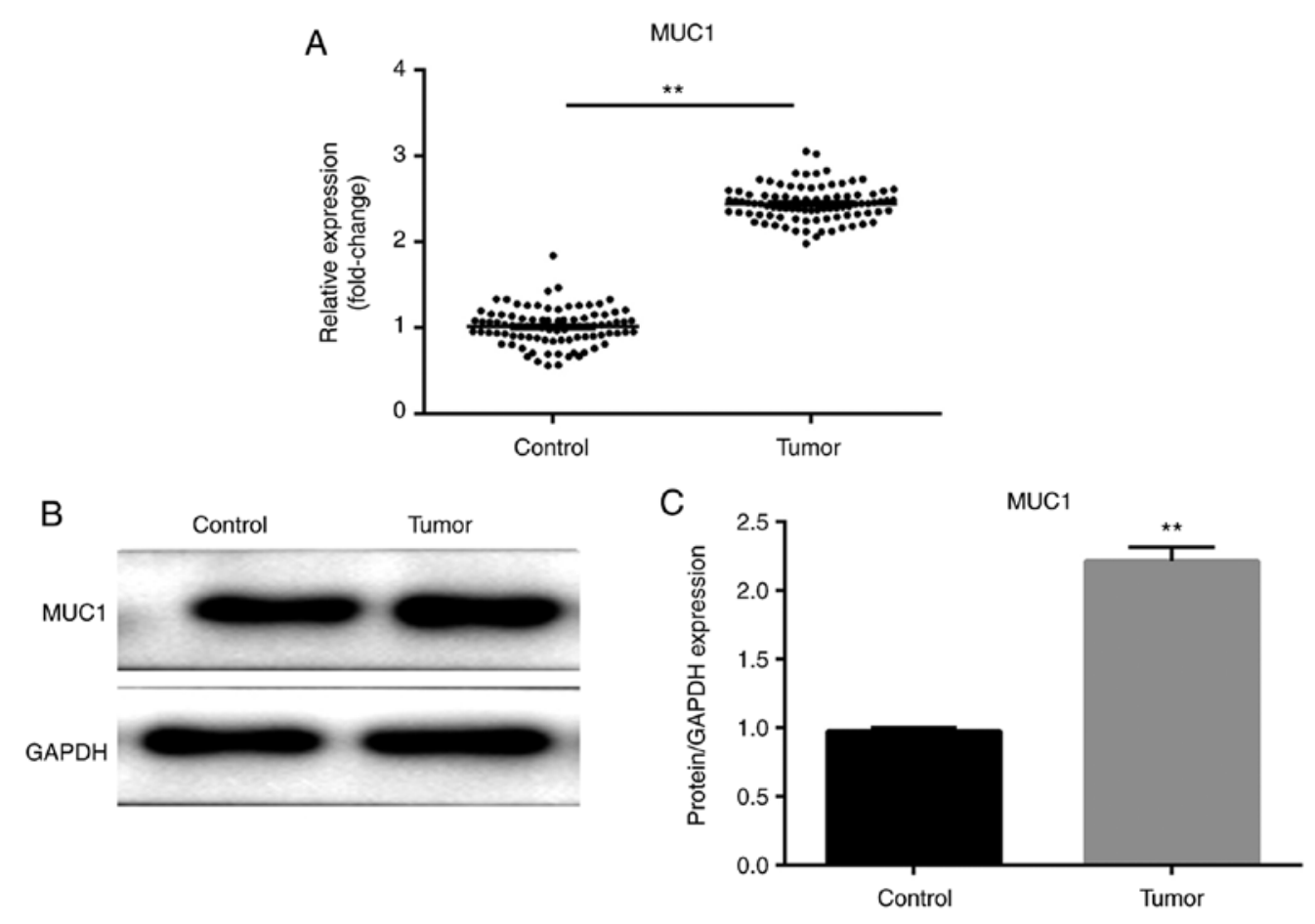

Figure 1. MUC1 was up-regulated in clinic NSCLC tissues. MUC1 mRNA and protein expressions in a total of 30 NSCLC tissues and matched adjacent normal tissues were examined by (A) RT-PCR and (B and C) western blot assays. The results were illustrated as the mean \pm SD of three independent experiments and each was performed in triplicate. ${ }^{* *} \mathrm{P}<0.01$ vs. control group.

no. 14161), BCL2 associated $X$ apoptosis regulator (Bax; cat. no. 2774), BCL2 apoptosis regulator (Bcl-2; cat. no. 2872), Caspase-3 (cat. no. 9662) and GAPDH (cat. no. 8884) at $4^{\circ} \mathrm{C}$ overnight. All antibodies were used at a 1:1,000 dilution and were purchased from Cell Signaling Technology, Inc. Membranes were washed three times with TBST. Following the primary incubation, membranes were incubated with secondary antibodies $(1: 1,000)$ for $2 \mathrm{~h}$ at $25^{\circ} \mathrm{C}$. Protein bands were visualized using an Enhanced Chemiluminescence Detection system. GAPDH was used as the loading control.

Statistical analysis. GraphPad Prism software (v5; GraphPad Software, Inc.) was used to perform all the statistical analysis. The Student's t-test was used to compare two groups and the one-way analysis of variance (ANOVA) followed by Tukey's test was used for the comparison of multiple groups. $\mathrm{P}<0.05$ was considered to indicate a statistically significant difference. All quantitative data are expressed as the mean \pm SD.

\section{Results}

MUC1 is upregulated in NSCLC tissues. RT-qPCR and western blotting were performed to detect the expression levels of MUC1 in NSCLC and adjacent noncancerous tissues. As shown in Fig. 1A-C, the mRNA and protein expression levels of MUC1 were upregulated in NSCLC tissues compared with adjacent noncancerous tissues $(\mathrm{P}<0.01)$, suggesting that $\mathrm{MUC1}$ may promote the progression and development of NSCLC.

MUC1 is upregulated in A549/PR cells. In order to further explore the role and underlying mechanisms of MUC1 in paclitaxel resistance in NSCLC, at $72 \mathrm{~h}$ post-transfection, the expression level of MUC1 was measured by RT-qPCR and western blot respectively. The mRNA expression level of MUC1 was significantly increased in A549/PR cells compared with A549 cells ( $\mathrm{P}<0.01$; Fig. 2A). The effect of MUC1 upregulation in NSCLC was further investigated by transfecting A549/PR cells with NC siRNA or MUC1 siRNA. The results revealed that the MUC1 siRNA significantly inhibited the mRNA and protein expression levels of MUC1 in A549/PR cells compared with the control $(\mathrm{P}<0.01$; Fig. 2B-D).

Silencing of MUC1 suppresses the proliferation of A549/PR cells following treatment with paclitaxel. Paclitaxel resistance in control and transfected cells A549/PR cells was investigated using the CCK-8 assay. As shown in Fig. 3A and B, the expression level of MUC1 was significantly decreased in MUC1-siRNA group and there was no significant difference between the proliferation rate of A549/PR cells and A549/PR cells transfected with NC siRNA following paclitaxel treatment. However, the proliferation rate of A549/PR cells transfected with MUC1 siRNA was significantly decreased compared with the untransfected cells and cells transfected with NC siRNA $(\mathrm{P}<0.01)$. These data suggested that the inhibition of MUC1 significantly enhanced paclitaxel sensitivity in A549/PR cells.

Inhibition of MUC1 promotes apoptosis of A549/PR cells following treatment with paclitaxel. The apoptotic rates of A549/PR cells transfected with NC siRNA or MUC1 siRNA following treatment with paclitaxel were determined using flow cytometry. There were no significant differences between the apoptotic rates of the untransfected cells and cells transfected with NC siRNA following treatment with paclitaxel $(\mathrm{P}<0.05$; 
A
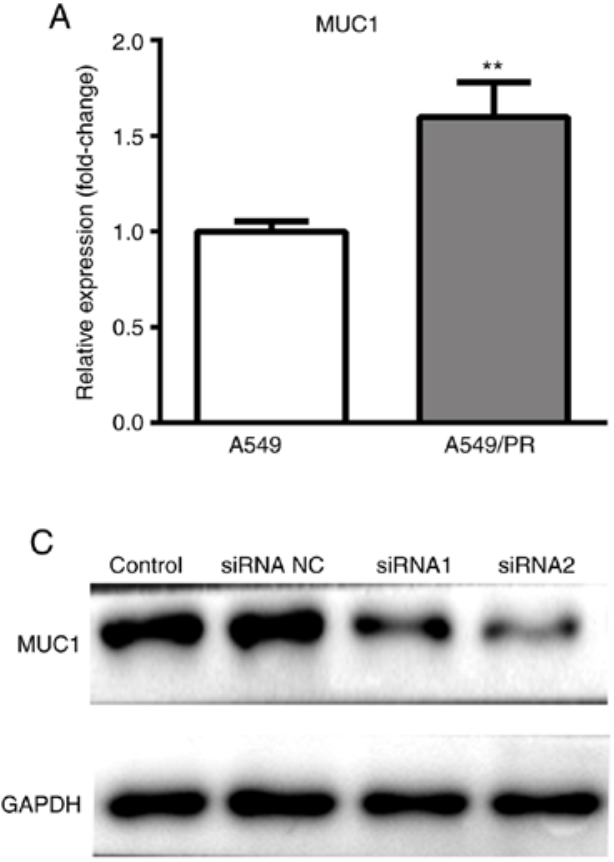

B
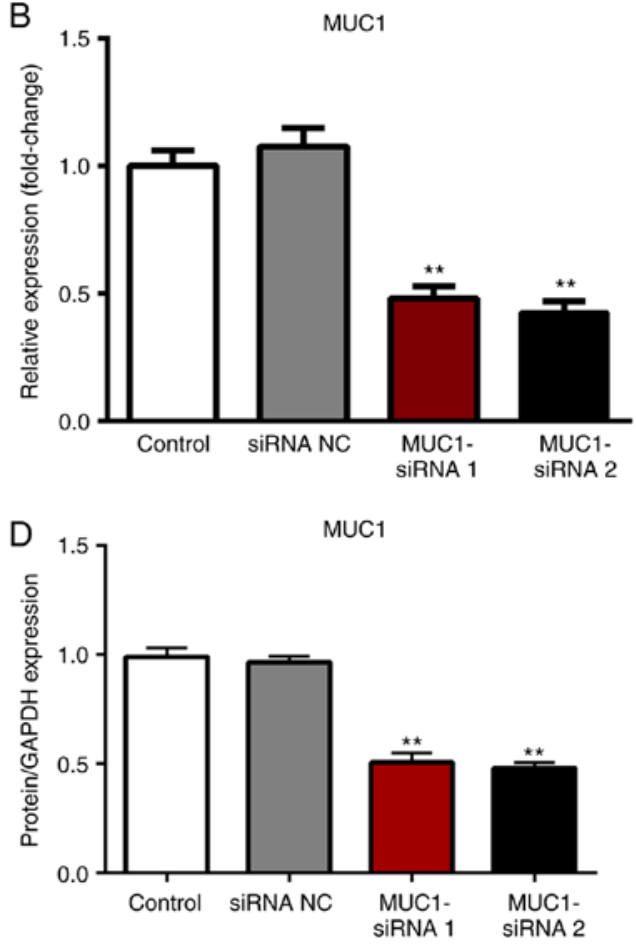

Figure 2. MUC1 was over-expressed in A549/PR cells. (A) MUC1 mRNA expression in A549/PR cells and A549 cells was examined by RT-PCR assay at 72 h post-transfection. ${ }^{* *} \mathrm{P}<0.01$ vs. A549 group. Transfection efficiency was assessed by (B) RT-PCR and (C and D) western blot assays at 72 h post-transfection with MUC1-siRNA in A549/PR cells. ${ }^{* *} \mathrm{P}<0.01$ vs. control or siRNA NC groups.

Fig. 4A and B). However, cells transfected with MUC1 siRNA exhibited significantly increased apoptosis compared with the untransfected cells or cells transfected with NC siRNA $(\mathrm{P}<0.01)$. The data suggested that inhibition of $\mathrm{MUC1}$ promoted apoptosis of paclitaxel-treated A549/PR cells.

MUC1 regulates the expression of Bcl2/BAX and caspase-3. Western blotting was performed to evaluate the protein expression levels of apoptosis-associated proteins in A549/PR cells transfected with NC siRNA or MUC1 siRNA following treatment with paclitaxel. The protein levels of Bax, Bcl-2 and caspase-3 were not significantly different between untransfected cells and cells transfected with NC siRNA following treatment with paclitaxel $(\mathrm{P}>0.05$; Fig. 5). The mRNA and protein levels of Bax and caspase- 3 were significantly upregulated while $\mathrm{Bcl}-2$ was significantly downregulated in cells transfected with MUC1 siRNA compared with untransfected cells or cells transfected with NC siRNA $(\mathrm{P}<0.01)$. The results further suggested that the inhibition of MUC1 promoted apoptosis of paclitaxel-treated A549/PR cells.

\section{Discussion}

The increase in environmental pollution and smoking in recent years has resulted in $>500,000$ new cases of lung cancer and $>400,000$ lung cancer-associated mortalities annually. Lung cancer has the highest incidence and mortality rates among all tumors. Approximately $\sim 80 \%$ of lung cancer cases are NSCLC. The majority of patients with NSCLC are diagnosed in the advanced stages of the disease and have inoperable tumors. Chemotherapy is therefore the main treatment strategy for patients with advanced NSCLC $(25,26)$. However,
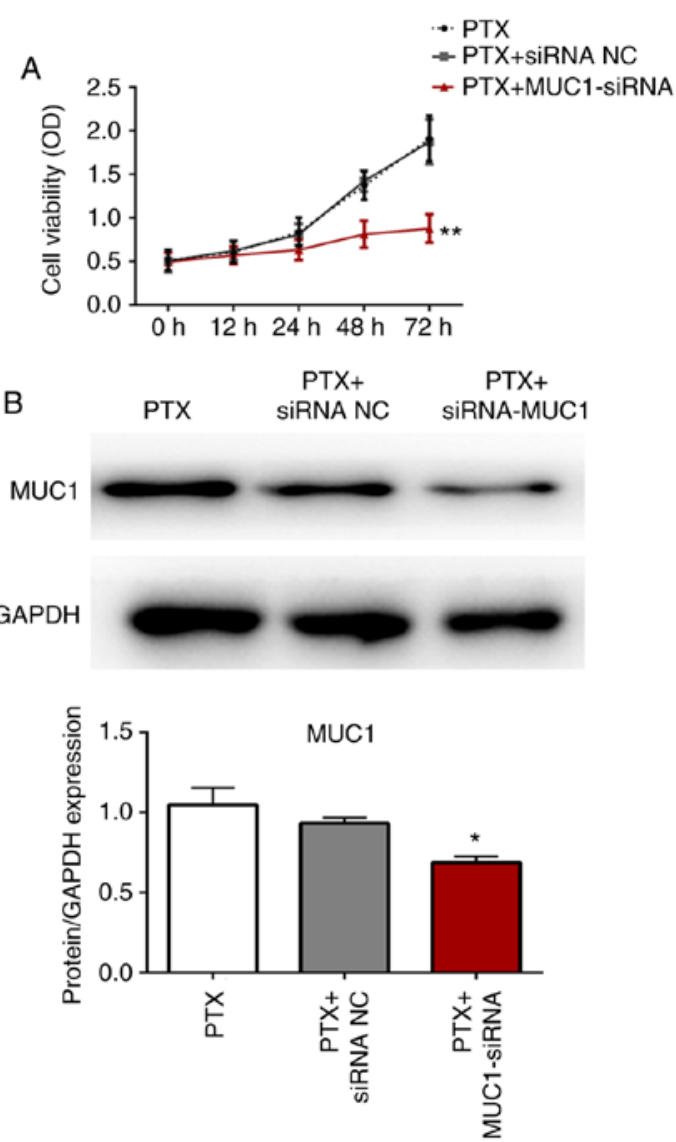

Figure 3. Silence of MUC1 suppressed the cell viability of A549/PR cells in treatment of paclitaxel. (A) The viabilities of A549/PR cells transfected with MUC1-siRNA for 0, 12, 24, 48 or $72 \mathrm{~h}$, respectively, were assessed by CCK-8 assay. ${ }^{* *} \mathrm{P}<0.01$ vs. PTX + siRNA NC group. (B) The expression level of MUC1 at $72 \mathrm{~h}$ post-transfection. ${ }^{*} \mathrm{P}<0.05$ vs. $\mathrm{PTX}+$ siRNA NC group. PTX, paclitaxel. 
A

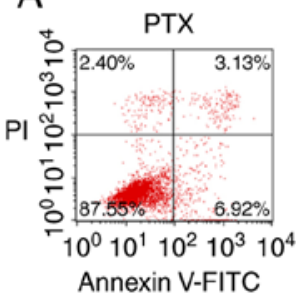

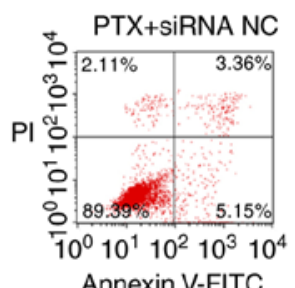

Annexin V-FITC
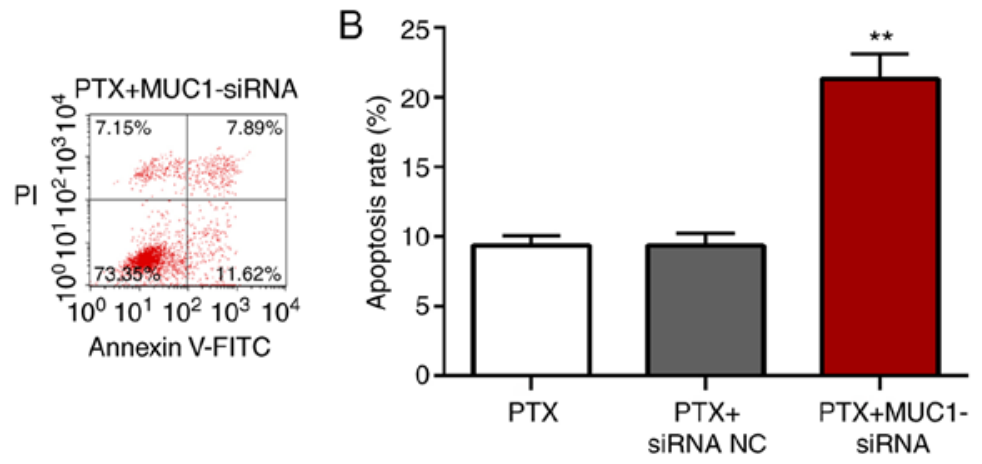

Figure 4. Inhibition of MUC1 promoted apoptosis of A549/PR cells in treatment of paclitaxel. (A) Apoptosis assays were performed by flow cytometry analysis after transfection with MUC1-siRNA for $48 \mathrm{~h}$ in A549/PR cells. (B) Quantified results of A were presented. ** P $<0.01$ vs. PTX + siRNA NC group. PTX, paclitaxel.
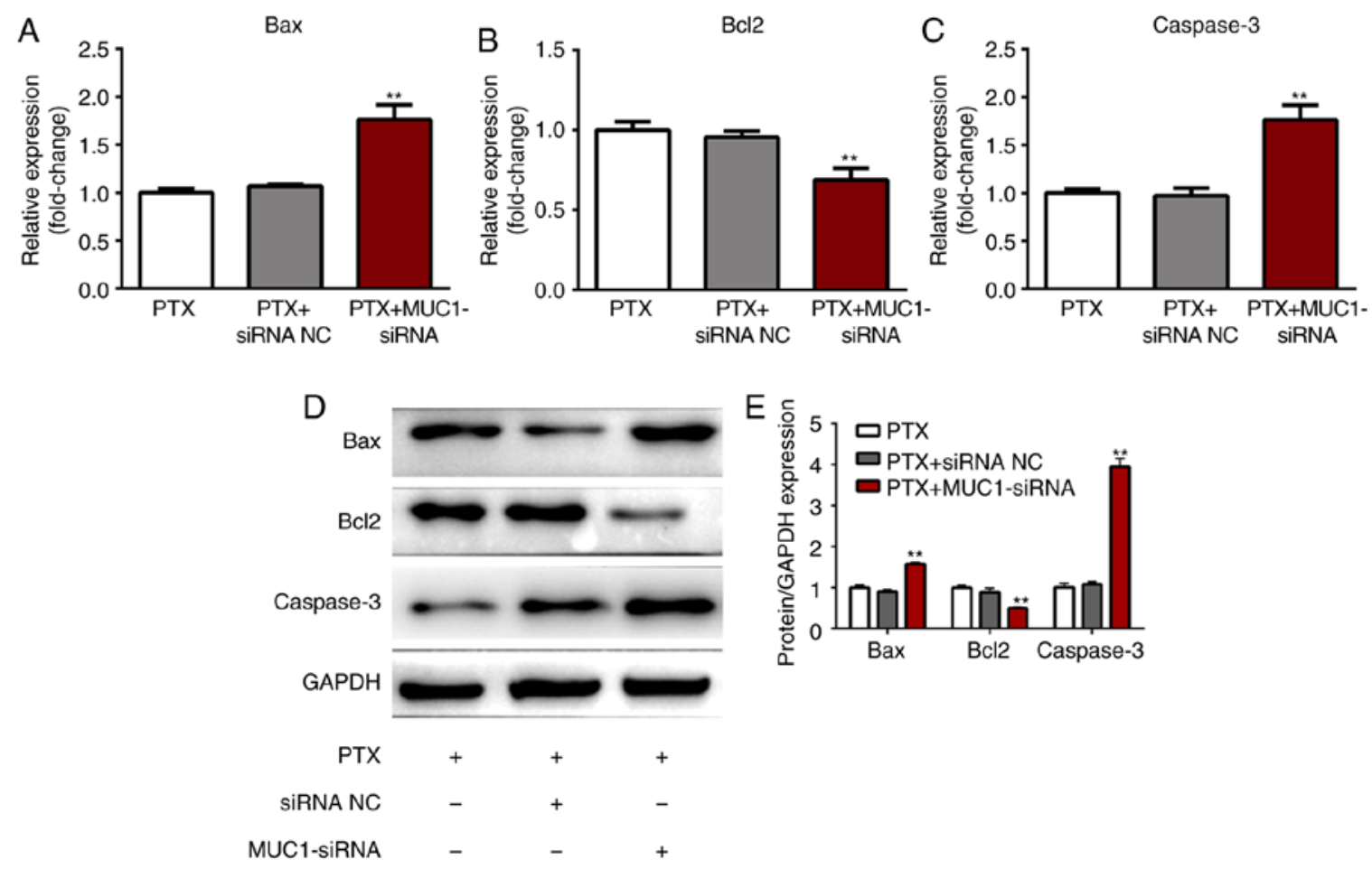

Figure 5. Downregulated MUC1 inhibited the expression of Bcl-2 and increased the expression of Bax and caspase-3. The expression of Bcl-2 in A549/PR cells transfected with MUC1-siRNA was significantly decreased both in (A-C) mRNA and (D and E) protein level, while the expression of Bax and caspase-3 was increased. ${ }^{* *} \mathrm{P}<0.01$ vs. PTX + siRNA NC group. PTX, paclitaxel; Bax, BCL2-associated X protein; Bcl2, B-cell lymphoma-2.

the efficacy rate of combined chemotherapy for NSCLC is $14-40 \%$, and may result in relapse. This phenomenon is closely associated with multidrug resistance in NSCLC (27). Drug resistance in tumors involves several mechanisms, and may lead to multidrug resistance $(28,29)$. Tumors with multidrug resistance are a common clinical problem, and often result in chemotherapy failure (27). Therefore, there has been increased interest in overcoming or preventing drug resistance. The main mechanisms of drug resistance in lung cancer include increased expression of multidrug resistance genes such as P-glycoprotein, glutathione transferases, decreased expression of topoisomerase and promoting DNA damage repair and anti-apoptotic ability of cancer cells (30).

Paclitaxel was originally isolated from the bark of Taxus pacificus and has cytotoxic effects on many types of cancer cells (31). Paclitaxel is a tubulin-binding drug and has exhib- ited better prospects; however, it is less cost-effective than other drugs (32). Additionally, paclitaxel is associated with drug resistance and cross-resistance in cancer cells. The mechanisms underlying paclitaxel resistance are complex and are not fully understood. Paclitaxel resistance may occur due to upregulation of P-glycoprotein, abnormal expression of microtubule regulatory proteins or post- translational changes expression of drug-resistant genes, abnormal signal transduction and cell death pathway regulation, and alterations in tubulin subtypes, proteins that regulate tubulin dynamics and paclitaxel binding sites (33). The elucidation of the pathways involved in paclitaxel resistance may allow the identification of suitable patients and guide the selection of treatment strategies. Furthermore, the emergence of drug resistance may be avoided or reversed, thereby improving the efficacy of chemotherapy. 
The mucin family consists of high molecular weight glycoproteins that protect and lubricate epithelial cells under physiological conditions. MUC1, a member of the mucin family, is a transmembrane heterodimer glycoprotein that is expressed at low levels in the proximal side of secretory epithelial cells in the mammary glands and respiratory, gastrointestinal and urogenital tracts. In epithelial tumors, MUC1 expression is upregulated and loses its polar distribution. Furthermore, MUC1 has been shown to serve an important role in tumor proliferation, invasion, metastasis, and chemotherapy resistance. MUC1 is highly expressed in $>90 \%$ of breast cancer cases and is associated with the occurrence and development of breast cancer by interacting with PI3K/AKT, MAP, NF- $\mathrm{B}$, Wnt, signal transducer and activator of transcription, tumor protein P53 and estrogen receptor (ER) $\alpha$ signaling pathways (34). Following the stimulation of breast cancer cells with EGF, the MUC1 intracellular segment was phosphorylated, CSK and MAPK signaling pathways were activated and cell proliferation was enhanced. In $17 \beta$-estradiol-stimulated breast cancer cells, MUC1 enhanced ER $\alpha$-mediated transcription and promoted the survival and growth of breast cancer cells. Ren et al (35), revealed that silencing MUC1 enhanced the sensitivity of A549 and ZR-75-1 cells to cytotoxic drugs. Horn et al (36), found that murine breast cancer DA3 cells overexpressing MUC1 increased the expression of interstitial markers, decreased the expression of epithelial markers and enhanced the ability of cells to produce extracellular matrix. In addition, previous studies have revealed that MUC1 was upregulated in NSCLC tumors, and that downregulation of MUC1 inhibited the progression of the disease $(37,38)$. In the future, we will conduct further experiments concerning the effect of MUC1 on paclitaxel resistance in $\mathrm{H} 1299$ or H1975 cell lines.

In the present study, MUC1 was upregulated in clinical NSCLC tissues and A549/PR cells. MUC1 silencing significantly suppressed the proliferation and promoted apoptosis of paclitaxel-treated A549/PR cells by regulating Bax, Bcl-2 and caspase-3 expression. The results obtained in the present study suggested that the modulation of MUC1 may serve as a promising therapeutic approach to overcome paclitaxel resistance in NSCLC.

\section{Acknowledgements}

Not applicable.

\section{Funding}

The present study was supported by Youth Innovation Fund of The General Hospital of Western Theater Command (grant no. 14732C119).

\section{Availability of data and materials}

The datasets used and/or analyzed during the present study are available from the corresponding author on reasonable request.

\section{Authors' contributions}

HYX, HG and HL designed the experiments and performed the statistical analysis. DL, WWY and LZ were involved in the study design, data acquisition and manuscript revision. PC, XMS, ZHL and GJW were in charge of writing the manuscript and data analysis. TZ collected all the samples and patients' clinical data. All authors read and approved the final manuscript.

\section{Ethics approval and consent to participate}

The present study was approved by the Ethics Committee of The General Hospital of Western Theater Command and all patients provided prior written informed consent.

\section{Patient consent for publication}

Not applicable.

\section{Competing interests}

The authors declare that they have no competing interests.

\section{References}

1. Islami F, Miller KD, Siegel RL, Fedewa SA, Ward EM and Jemal A: Disparities in liver cancer occurrence in the United States by race/ethnicity and state. CA Cancer J Clin 67: 273-289, 2017.

2. Breathnach OS, Freidlin B, Conley B, Green MR, Johnson DH, Gandara DR, O'Connell M, Shepherd FA and Johnson BE: Twenty-two years of phase III trials for patients with advanced non-small-cell lung cancer: Sobering results. J Clin Oncol 19: 1734-1742, 2001.

3. Reck M,Heigener DF, Mok T, Soria JC and Rabe KF: Management of non-small-cell lung cancer: Recent developments. Lancet 382: 709-719, 2013.

4. Cufer T, Ovcaricek T and O'Brien ME: Systemic therapy of advanced non-small cell lung cancer: Major-developments of the last 5-years. Eur J Cancer 49: 1216-1225, 2013.

5. Yasukawa M, Sawabata N, Kawaguchi T, Kawai N, Nakai T, Ohbayashi C and Taniguchi S: Histological grade: Analysis of prognosis of non-small cell lung cancer after complete resection. In Vivo 32: 1505-1512, 2018.

6. Wang L, Shang X and Feng Q: LncRNA TATDN1 contributes to the cisplatin resistance of non-small cell lung cancer through TATDN1/miR-451/TRIM66 axis. Cancer Biol Ther 20: 261-271, 2019.

7. Roach MC, Bradley JD and Robinson CG: Optimizing radiation dose and fractionation for the definitive treatment of locally advanced non-small cell lung cancer. J Thorac Dis 10 (Suppl 21): S2465-S2473, 2018.

8. Chu T, Jiang L, Ying W and Han B: M30/M65 ratio predicts the outcome of paclitaxel chemotherapy for NSCLC. Clin Transl Oncol 19: 326-331, 2017.

9. Wu YL, Saijo N, Thongprasert S, Yang JC, Han B, Margono B, Chewaskulyong B, Sunpaweravong P, Ohe Y, Ichinose Y, et al: Efficacy according to blind independent central review: Post-hoc analyses from the phase III, randomized, multicenter, IPASS study of first-line gefitinib versus carboplatin/paclitaxel in Asian patients with EGFR mutation-positive advanced NSCLC. Lung Cancer 104: 119-125, 2017.

10. Lu Y, Wang J, Liu L, Yu L, Zhao N, Zhou X and Lu X: Curcumin increases the sensitivity of Paclitaxel-resistant NSCLC cells to Paclitaxel through microRNA-30c-mediated MTA1 reduction. Tumour Biol: doi:10.1177/1010428317698353.

11. Kubo N, Noda SE, Takahashi A, Yoshida Y, Oike T, Murata K, Musha A, Suzuki Y, Ohno T, Takahashi T, et al: Radiosensitizing effect of carboplatin and paclitaxel to carbon-ion beam irradiation in the non-small-cell lung cancer cell line H460. J Radiat Res (Tokyo) 56: 229-238, 2015.

12. Ding J, Li M, Deng L and Li T: Study on biological characteristics and mechanism of paclitaxel induced drug resistance in endometrial carcinoma cells. BioMed Res Int 2018: 8372085, 2018.

13. Sobue S, Mizutani N, Aoyama Y, Kawamoto Y, Suzuki M, Nozawa Y, Ichihara $\mathrm{M}$ and Murate T: Mechanism of paclitaxel resistance in a human prostate cancer cell line, PC3-PR, and its sensitization by cabazitaxel. Biochem Biophys Res Commun 479: 808-813, 2016. 
14. Tsakonas G, De Petris L and Ekman S: Management of brain metastasized non-small cell lung cancer (NSCLC) - From local treatment to new systemic therapies. Cancer Treat Rev 54: 122-131, 2017.

15. Zhang H, Liu Q, Kong L and Xu S: Mucin 1 downregulation impairs the anti-necroptotic effects of glucocorticoids in human bronchial epithelial cells. Life Sci 221: 168-177, 2019.

16. Song ZB, Gao SS, Yi XN, Li YJ, Wang QM, Zhuang ZH and Wang LD: Expression of MUC1 in esophageal squamous-cell carcinoma and its relationship with prognosis of patients from Linzhou city, a high incidence area of northern China. World J Gastroenterol 9: 404-407, 2003.

17. Sakamoto H, Yonezawa S, Utsunomiya T, Tanaka S, Kim YS and Sato E: Mucin antigen expression in gastric carcinomas of young and old adults. Hum Pathol 28: 1056-1065, 1997.

18. Wreesmann VB, Sieczka EM, Socci ND, Hezel M, Belbin TJ, Childs G, Patel SG, Patel KN, Tallini G, Prystowsky M, et al: Genome-wide profiling of papillary thyroid cancer identifies MUC1 as an independent prognostic marker. Cancer Res 64 3780-3789, 2004.

19. Morari EC, Silva JR, Guilhen AC, Cunha LL, Marcello MA, Soares FA, Vassallo J and Ward LS: Muc-1 expression may help characterize thyroid nodules but does not predict patients outcome. Endocr Pathol 21: 242-249, 2010.

20. Abrosimov A, Saenko V, Meirmanov S, Nakashima M Rogounovitch T, Shkurko O, Lushnikov E, Mitsutake N, Namba H and Yamashita S: The cytoplasmic expression of MUC1 in papillary thyroid carcinoma of different histological variants and its correlation with cyclin D1 overexpression. Endocr Pathol 18: 68-75, 2007.

21. Nabavinia MS, Gholoobi A, Charbgoo F, Nabavinia M,Ramezani M and Abnous K: Anti-MUC1 aptamer: A potential opportunity for cancer treatment. Med Res Rev 37: 1518-1539, 2017.

22. Sun ZG, Zhang M, Yang F, Gao W, Wang Z and Zhu LM: Clinical and prognostic significance of signal transducer and activator of transcription 3 and mucin 1 in patients with non-small cell lung cancer following surgery. Oncol Lett 15: 4278-4288, 2018.

23. Xu T, Li D, Wang H, Zheng T, Wang G and Xin Y: MUC1 downregulation inhibits non-small cell lung cancer progression in human cell lines. Exp Ther Med 14: 4443-4447, 2017.

24. Sun H, Zhou X, Bao Y, Xiong G, Cui Y and Zhou H: Involvement of miR-4262 in paclitaxel resistance through the regulation of PTEN in non-small cell lung cancer. Open Biol 9: 180227, 2019.

25. Bocci G, Di Paolo A and Danesi R: The pharmacological bases of the antiangiogenic activity of paclitaxel. Angiogenesis 16 481-492, 2013
26. Milane L, Duan Z and Amiji M: Development of EGFR-targeted polymer blend nanocarriers for combination paclitaxel/lonidamine delivery to treat multi-drug resistance in human breast and ovarian tumor cells. Mol Pharm 8: 185-203, 2011.

27. Wang L, Liu X, Ren Y, Zhang J, Chen J, Zhou W, Guo W, Wang X, Chen H, Li M, et al: Cisplatin-enriching cancer stem cells confer multidrug resistance in non-small cell lung cancer via enhancing TRIB1/HDAC activity. Cell Death Dis 8: e2746, 2017.

28. Lehnert M: Clinical multidrug resistance in cancer: A multifactorial problem. Eur J Cancer 32A: 912-920, 1996.

29. Gottesman MM and Pastan I: Biochemistry of multidrug resistance mediated by the multidrug transporter. Annu Rev Biochem 62: 385-427, 1993

30. Volm M, Koomägi R, Efferth T and Mattern J: Protein expression profiles of non-small cell lung carcinomas: Correlation with histological subtype. Anticancer Res 22: 2321-2324, 2002.

31. Wani MC, Taylor HL, Wall ME, Coggon P and McPhail AT: Plant antitumor agents. VI. The isolation and structure of taxol, a novel antileukemic and antitumor agent from Taxus brevifolia. J Am Chem Soc 93: 2325-2327, 1971.

32. Waud WR, Gilbert KS, Harrison SD Jr and Griswold DP Jr: Cross-resistance of drug-resistant murine P388 leukemias to taxol in vivo. Cancer Chemother Pharmacol 31: 255-257, 1992.

33. Morin PJ: Drug resistance and the microenvironment: Nature and nurture. Drug Resist Updat 6: 169-172, 2003.

34. Kufe DW: MUC1-C oncoprotein as a target in breast cancer: Activation of signaling pathways and therapeutic approaches. Oncogene 32: 1073-1081, 2013.

35. Ren J, Agata N, Chen D, Li Y, Yu WH, Huang L, Raina D, Chen W, Kharbanda S and Kufe D: Human MUC1 carcinoma-associated protein confers resistance to genotoxic anticancer agents. Cancer Cell 5: 163-175, 2004.

36. Horn G, Gaziel A, Wreschner DH, Smorodinsky NI and Ehrlich M: ERK and PI3K regulate different aspects of the epithelial to mesenchymal transition of mammary tumor cells induced by truncated MUC1. Exp Cell Res 315: 1490-1504, 2009.

37. Wei X, Lai Y, Li J, Qin L, Xu Y, Zhao R, Li B, Lin S, Wang S, Wu Q, et al: PSCA and MUC1 in non-small-cell lung cancer as targets of chimeric antigen receptor T cells. OncoImmunology 6: e1284722, 2017.

38. Bouillez A, Adeegbe D, Jin C, Hu X, Tagde A, Alam M, Rajabi H, Wong KK and Kufe D: MUC1-C promotes the suppressive immune microenvironment in non-small cell lung cancer. OncoImmunology 6: e1338998, 2017. 